\title{
Swyer syndrome with malignant germ cell tumor: a case report
}

\author{
Soha Talaat Hamed and MennatAllah Mohamed Hanafy * (D)
}

\begin{abstract}
Background: Swyer syndrome (Pure gonadal dysgenesis, $46 \mathrm{XY}$ ) is a rare form of disorder of sexual development. These patients presented with external female phenotype, normal Mullerian structures and streak gonads. Pure gonadal dysgenesis, $X Y$ patients are more likely to develop germ cell tumors due to the presence of the $Y$ chromosome.

Case presentation: A 19-year-old patient with a female external phenotype presented with primary amenorrhea. Clinical examination, Karyotyping, imaging, and histopathological assessment revealed Swyer syndrome. On imaging, a right adnexal mass with calcification was detected. Laparoscopic surgery with histopathology revealed a malignant germ cell tumor.

Conclusions: Swyer syndrome represents a rare form of sexual development that necessitates a meticulous clinical, laboratory and radiological evaluation. Clinically, the patients have a female external phenotype with 46xy Karyotyping. Imaging, Ultrasound is the primary imaging modality Imaging and MRI helps in detection of the exact site of streak gonads and characterization of lesions. CT is useful in detecting calcification, which is a hallmark in the diagnosis of gonadoblastoma. Early diagnosis of Swyer syndrome is crucial as prophylactic gonadectomy in these cases reduces the risk of developing germ cell tumors.
\end{abstract}

Keywords: Swyer syndrome, Gonadal dysgenesis, Germ cell tumors

\section{Background}

Swyer syndrome (Pure gonadal dysgenesis, $46 \mathrm{XY}$ ) is a rare form of disorders of sexual development [1]. Swyer syndrome results from abnormal intrauterine sexual differentiation [2]. The early stages of testicular formation in intrauterine life necessitate the presence of the $\mathrm{Y}$ chromosome with several genes on it, the most important of which is SRY (the sex-determining region of the $\mathrm{Y}$ chromosome) [3]. Swyer syndrome is usually associated with the mutation of these genes [3, 4]. The absence of the testis leads to the absence of testosterone and AntiMullerian hormone [4]. So these patients have phenotypically female external genitalia with internal Mullerian duct structures but no Wolffian duct structures (internal

*Correspondence: drmennamhanafy@gmail.com

Department of Diagnostic and Interventional Radiology, Faculty

of Medicine, Cairo University, 1 kasrElainy street FomElkalig, Cairo, Egypt male organs). The upper part of the vagina and tubes are normal or reduced in size, and the uterus is small or rudimentary. The gonads are non-functioning dysgenetic streaks [2].

Pure gonadal dysgenesis, $\mathrm{XY}$ patients show an increased incidence of germ cell tumors due to the presence of the $\mathrm{Y}$ chromosome. The most frequent of these germ cell tumors are gonadoblastoma and Dysgerminoma [5].

It is a challenge to diagnose Swyer syndrome due to complex clinical, laboratory and imaging findings. However, early diagnosis and proper management would diminish the risk of developing germ cell tumors.

We diagnosed a rare case of pure gonadal dysgenesis $\mathrm{XY}$ (Swyer syndrome) with a malignant germ cell tumor. 


\section{Case presentation}

In January 2020, we report a 19-year-old patient with a female external phenotype presented to us with primary amenorrhea. No significant family history was present. No history of abdominal pain, diarrhea, constipation or urinary symptoms. On clinical examination, she was about $168 \mathrm{~cm}$ in height and $67 \mathrm{~kg}$ with female external genitalia and small breasts. Axillary and pubic hair was sparse. No palpable masses could be appreciated.

Pelvic ultrasound revealed a small infantile uterus measuring about $4.4 \times 1.2 \mathrm{~cm}$ with non-visualized ovaries, yet a right adnexal solid complex mass was noted. This right adnexal solid lesion measured about $6.5 \times 3.5 \mathrm{~cm}$ in maximum dimensions seen abutting the urinary bladder. The right adnexal lesion had two inseparable parts; the superior one elicited mixed echogenicity mainly isoechoic with peripheral hyperechoic foci, likely representing calcifications and the inferior one appeared hypoechoic with posterior shadowing. On color Doppler, there was increased vascularity within the right adnexal lesion as shown in Fig. 1.

Pelvic MRI examination confirmed the presence of an infantile uterus with a right adnexal lesion indenting the urinary bladder and rectum but no evidence of invasion. This right adnexal lesion had two inseparable parts; the anterosuperior one elicited isointense signal in T1 and T2 and the posteroinferior one elicited hypointense signal in all pulse sequences as shown in Fig. 2. There was no evidence of lymphadenopathy.

Complementary non-contrast CT confirmed the presence of a densely calcified portion of the right adnexal lesion. While the rest of the right adnexal lesion appeared to have soft-tissue attenuation with incomplete marginal calcifications as shown in Fig. 3.

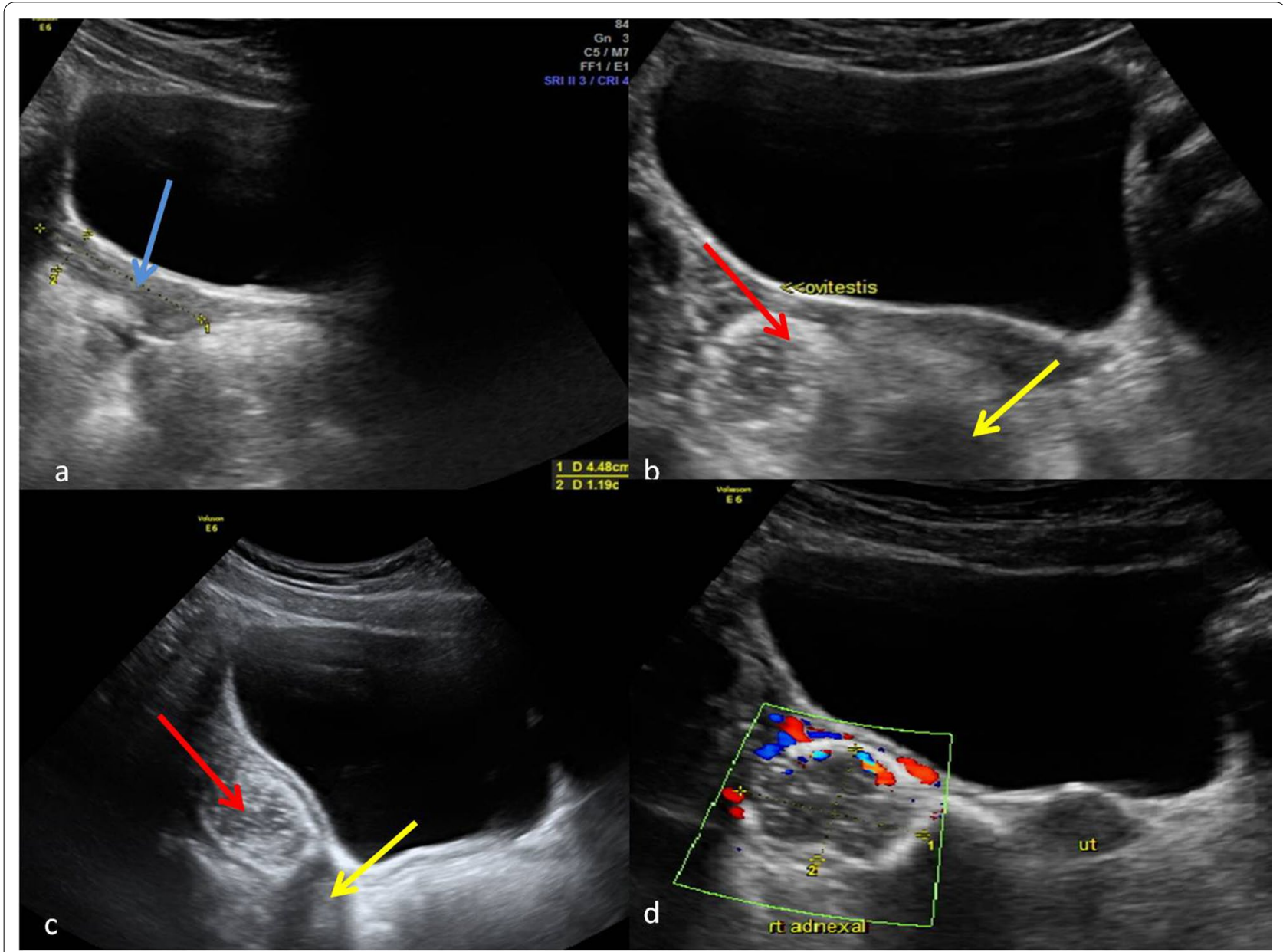

Fig. 1 Pelvic ultrasound revealed an infantile uterus (blue arrow) (a) with a right adnexal solid lesion showing apparently two parts; the superior one (red arrow) elicited mixed echogenicity mainly isoechoic and the inferior one (yellow arrow) was hypoechoic with posterior shadowing (b, c). It displayed increased vascularity on color Doppler $(\mathbf{d})$ 


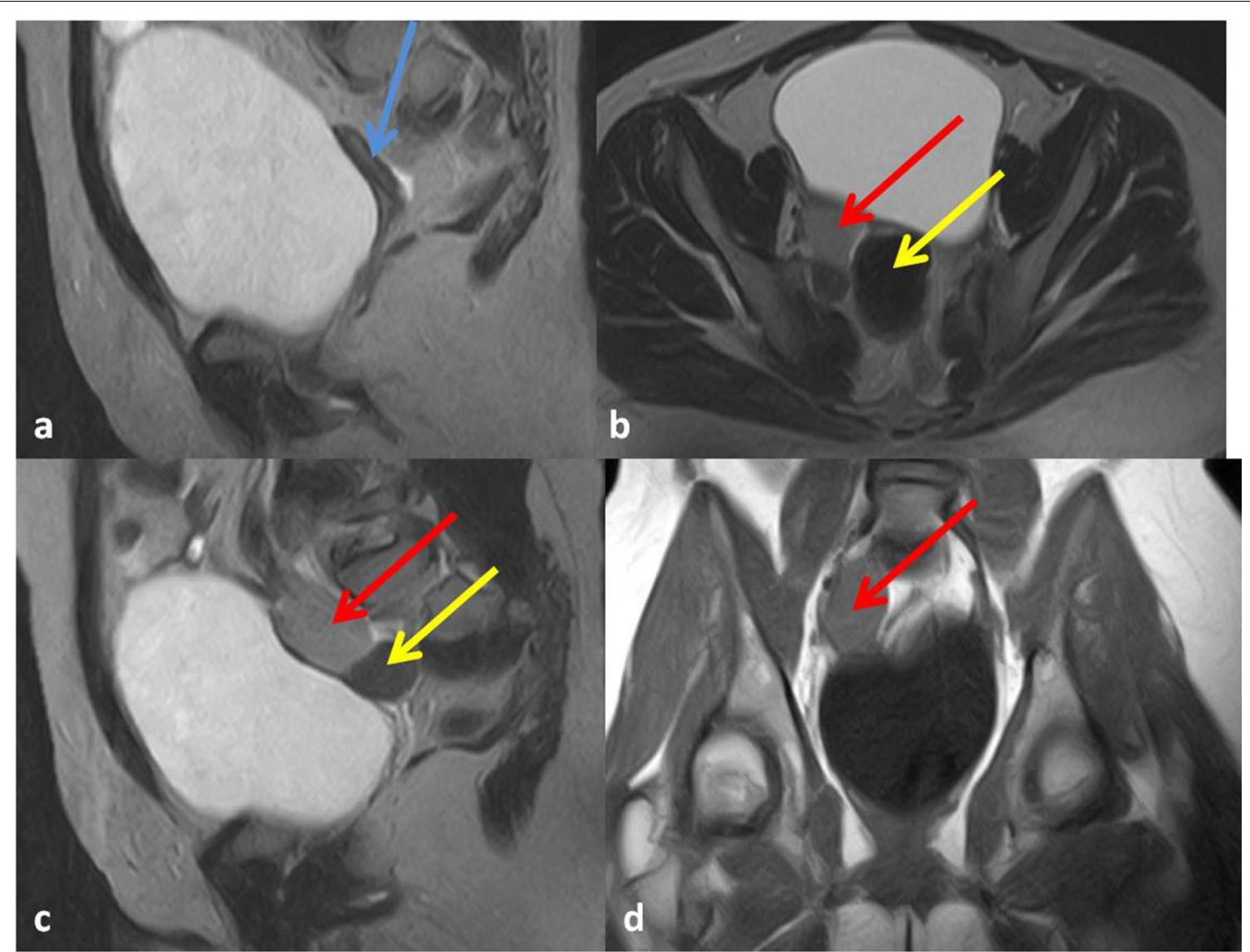

Fig. 2 Pelvic MRI examination confirmed the presence of an infantile uterus(blue arrow) (a) with a right adnexal lesion that showed two parts; the anterosuperior part (red arrow) elicited isointense signal in $\mathrm{T} 1$ and $\mathrm{T} 2$ and the posteroinferior one (yellow arrow) elicited hypointense signal in all pulse sequences $(\mathbf{b}, \mathbf{c}, \mathbf{d})$

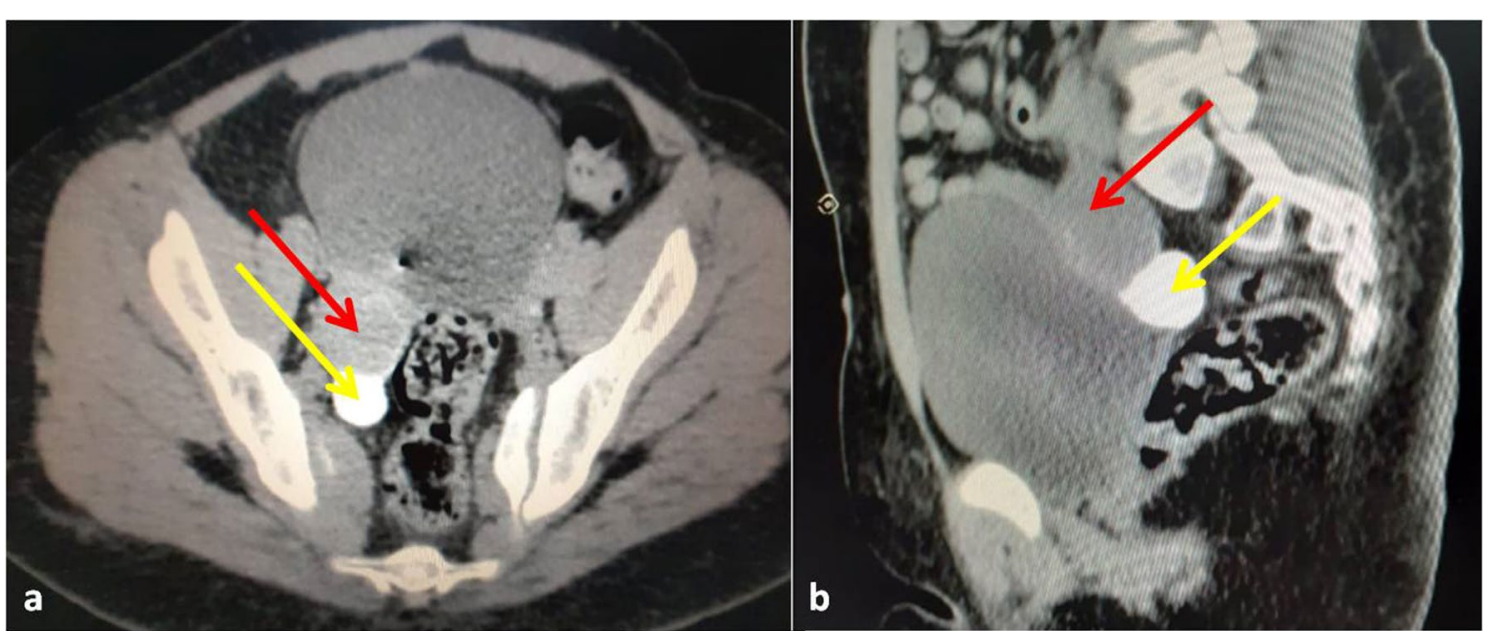

Fig. 3 Complementary CT confirmed the presence of a right adnexal calcified part (yellow arrow). The anterosuperior part of the right adnexal lesion appeared to have soft-tissue attenuation with faint incomplete marginal calcifications (red arrow) 
Karyotyping revealed a genotype $46, \mathrm{XY}$ as shown in Fig. 4. Low testosterone levels were discovered during laboratory investigation.

Laparoscopic excision of the right adnexal lesion and histopathology assessment revealed malignant germ cell tumor dysgerminoma complicating gonadoblastoma.

\section{Discussion}

Swyer syndrome (pure gonadal dysgenesis, $\mathrm{XY}$ ) represents a rare form of disorder of sexual development that was first described by Jim Swyer in 1955 [4]. It has an incidence of about 1:80,000 [2]. The etiology of Swyer syndrome is thought to be due to genetic mutation of genes required for testicular formation. It is usually caused by a mutation in the SRY gene due to deletion in the DNA-binding region of the SRY gene. But it may be due to mutation in other genes that leads to inhibition of SRY function or mutation of SRY function $[6,7]$.

Swyer syndrome is usually presented at the time of puberty and adolescence with primary amenorrhea. This syndrome is characterized by phenotypically female with female external genitalia, gonadal dysgenesis with bilateral streak gonads and Mullerian structures (small or rudimentary uterus, fallopian tube, vagina). On karyotyping, $46 \mathrm{XY}$ is detected $[2,5,8]$.
The differential diagnosis of Swyer syndrome (Pure gonadal dysgenesis, XY) includes Mayer-RokitanskyKüster-Hauser syndrome (XX), which is a common cause of primary amenorrhea [2]. This syndrome has an incidence of one in every 5000 live females [9]. It is characterized by variable degrees of Müllerian duct aplasia with aplasia of the vagina and a rudimentary or absent uterus. But on the contrary to Swyer syndrome, they have normal secondary sex characteristics and a normal female karyotype $(46, \mathrm{XX})[9,10]$.

Swyer syndrome has another differential diagnosis: Congenital Androgenic Insensitivity Syndrome. In Congenital Androgenic Insensitivity Syndrome, the karyotype is $\mathrm{XY}$ with high testosterone level. These patients have a female phenotype with normal, yet undescended testis present, absent Mullerian structures and normal breast development $[4,11]$. On the other hand, the breasts are usually underdeveloped with low testosterone levels in Swyer syndrome as in our case.

Also, because true hermaphrodites can present with a female phenotype, they are a differential diagnosis for Swyer syndrome. True hermaphrodite patients, on the other hand, may have ovotestes or a combination of an ovary and testes [6].

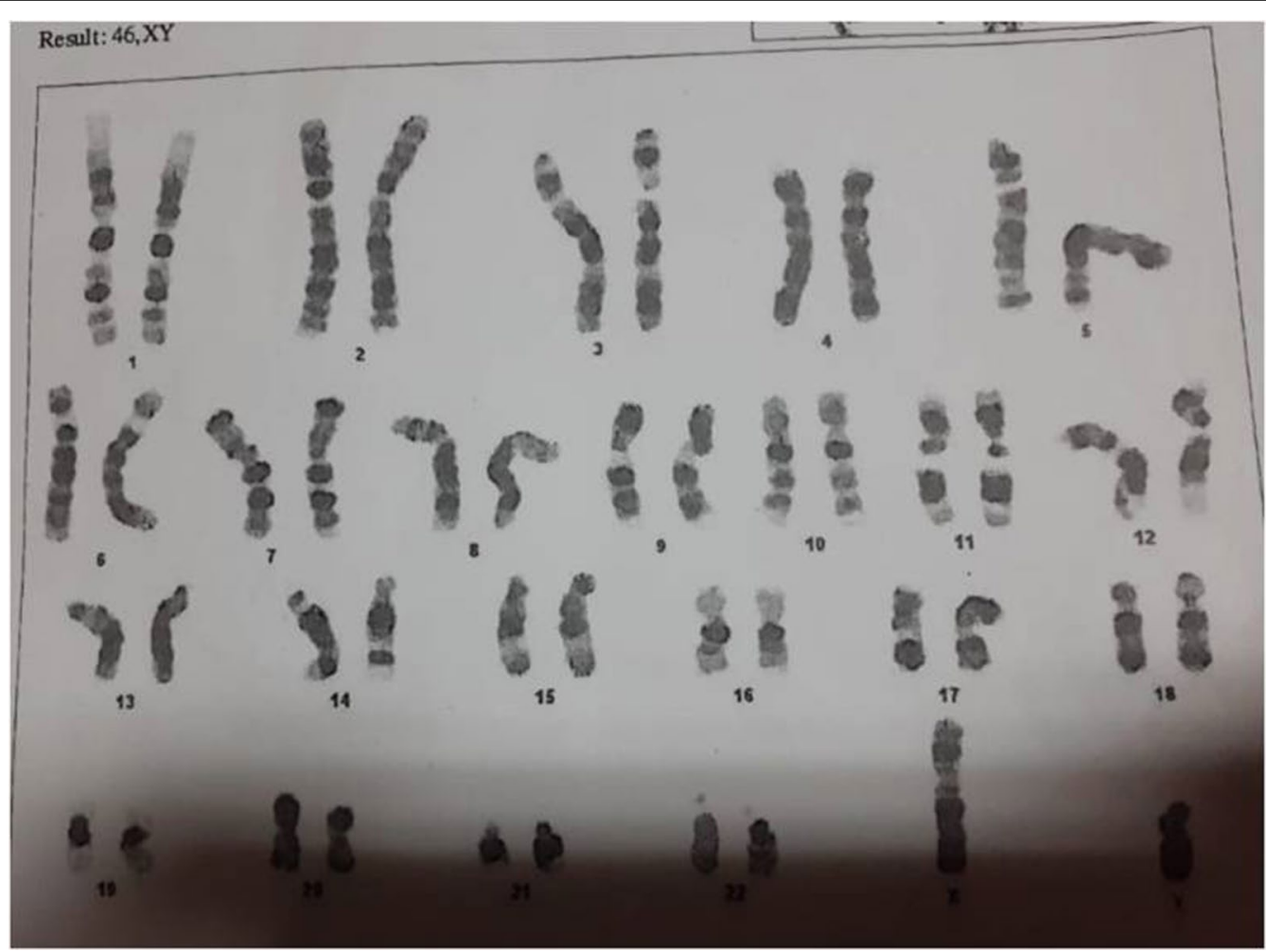

Fig. 4 Karyotyping of the patient revealed male genotype 46xy 
Because of the presence of the $Y$ chromosome, patients with pure gonadal dysgenesis $\mathrm{XY}$ are at a higher risk of developing germ cell neoplasms. Gonadoblastoma and dysgerminoma are the most common $[6,8]$. As a result, in Swyer syndrome, bilateral prophylactic gonadectomy is considered to reduce the risk of developing germ cell neoplasm [6].

Gonadoblastoma is an uncommon germ cell-sex cordstromal tumor. They are associated with disorders of sexual development. Gonadoblastoma is a mixed germ cell tumor with undifferentiated cells. This makes it a precursor to seminoma/dysgerminoma tumors [12]. The gonadoblastomas are associated with dysgerminoma in $50-60 \%$ of cases and with other malignant germ cell tumors like yolk sac tumor, embryonal carcinoma, and choriocarcinoma in $10 \%$ of cases [13].

Dysgerminoma is a malignant tumor that arises from the primordial germ cells identical to testicular seminoma $[5,12]$. Imaging findings of Dysgerminoma include multilobulated solid masses with prominent fibrovascular septa and speckled calcifications [5, 14].

In our case CT confirms the presence of calcification that was suspected in the ultrasound. Raafey et al. described a case of bilateral gonadoblastoma with dysgerminoma that was manifested by bilateral adnexal lesions with calcifications [15].

\section{Conclusions}

Swyer syndrome represents a rare form of sexual development that necessitates a meticulous clinical, laboratory and radiological evaluation. Clinically, the patients have a female external phenotype with 46xy Karyotyping. Imaging, Ultrasound is the primary imaging modality Imaging and MRI helps in detection of the exact site of streak gonads and characterization of lesions. CT is useful in detecting calcification, which is a hallmark in the diagnosis of gonadoblastoma. Early diagnosis of Swyer syndrome is crucial as prophylactic gonadectomy in these cases reduces the risk of developing germ cell tumors.

\section{Abbreviations}

$\mathrm{CT}$ : Computed tomography; MRI: Magnetic resonance imaging.

\section{Acknowledgements}

Not applicable.

\section{Authors' contributions}

ST and MH wrote the manuscript and collected patient data. $\mathrm{MH}$ is responsible for correspondence to journal. All authors read and approved the final manuscript

Funding

No funding sources.

\section{Availability of data and material}

The datasets used and analysed are available from the corresponding author on reasonable request.

\section{Declarations}

\section{Ethics approval and consent to participate}

The study was approved by the ethical committee of Radiology department; faculty of medicine, Cairo University, the ethical approval number is not available. An informed consent was taken.

\section{Consent for publication}

The patient included in this research gave written consent to publish the data contained within this study.

\section{Competing interests}

The authors declare no competing interests.

Received: 7 May 2021 Accepted: 1 September 2021

Published online: 28 September 2021

\section{References}

1. Mayur P, Parikshaa G, Anil B et al (2019) 'Size does matter prophylactic gonadectomy in a case of Swyer syndrome. J Gynecol Obstet Hum Reprod 48(4):283-286

2. Da Silva RS, Monteiro IC, Braz Dos Santos LG et al (2015) A case of swyer syndrome associated with advanced gonadal dysgerminoma involving long survival. Case Rep Oncol 8(1):179-184

3. Agarwal A, Agarwal S (2017) Swyer syndrome with gonadoblastoma: a clinicoradiological approach. J Hum Reprod 10(1):65-68

4. Meyer KF, Freitas Filho LG, Silva KI, Trauzcinsky PA, Reuter C, Souza MBM (2019) The XY female and SWYER syndrome. Urol Case Rep. 26:100939

5. Yadav P, Khaladkar S, Gujrati A (2016) Imaging findings in dysgerminoma in a case of $46 \mathrm{XY}$, complete gonadal dysgenesis (Swyer syndrome). J Clin Diagn Res 10(9):TD10-TD12

6. Han $Y$, Wang $Y, L i$ Q et al (2011) Dysgerminoma in a case of $46, X Y$ pure gonadal dysgenesis (swyer syndrome): a case report. Diagn Pathol 6:84

7. Goyal M, Batra S, Singh P et al (2019) Syndrome with heterotopic adrenal cortical tissue in streak gonads: a rare case and review of literature. J Hum Reprod Sci 12(4):345-347

8. Chand MT, Turner S, Solomon LA et al (2020) A case of $45, X / 46, X Y$ mosaicism presenting as swyer syndrome. J Pediatr Adolesc Gynecol 33(5):577-580

9. Herlin MK, Petersen MB, Brännström M (2020) Mayer-Rokitansky-KüsterHauser (MRKH) syndrome: a comprehensive update. Orphanet J Rare Dis 15:214

10. Fontana L, Gentilin B, Fedele L et al (2017) Genetics of Mayer-RokitanskyKüster-Hauser (MRKH) syndrome. Clin Genet 91(2):233-246

11. Culha C, Ozkaya M, Serter R et al (2012) Swyer's syndrome: in a fifty-yearold female. J Obstet Gynecol India 62(5):571-574

12. Piazza MJ, Urbanetz AA (2019) Germ cell tumors in dysgenetic gonads. Clinics (Sao Paulo) 74:e408

13. Kafil A, Sadaf H, Durre A (2018) Recurrent unilateral gonadoblastomaa rare histopathological presentation. J Surg Med. https://doi.org/10. 28982/josam.407263

14. Zhao S, Sun F, Bao L et al (2020) Pure dysgerminoma of the ovary: CT and MRI features with pathological correlation in 13 tumors. J Ovarian Res 13:71

15. Raafey MA, Abdulwaasey M, Fatima SS et al (2020) Bilateral gonadoblastoma with dysgerminoma in a phenotypically normal female with 46XX karyotype: report of a rare case and literature review. Cureus 12(7):e8990. https://doi.org/10.7759/cureus.8990

\section{Publisher's Note}

Springer Nature remains neutral with regard to jurisdictional claims in published maps and institutional affiliations. 